\title{
Time matters greatly in acute stroke care
}

\author{
Michelle P. Lin \\ Department of Neurology, Mayo Clinic Florida, Jacksonville, Florida, United States
}

\begin{abstract}
Stroke is a leading cause of morbidity worldwide. 'Time is brain' is a slogan that characterises contemporary stroke care. Rapid evaluation of stroke begins with community education to better recognise the signs and symptoms of stroke, an organised system of care by first responders and the emergency department, prompt evaluation by the stroke team, and the timely administration of reperfusion including intravenous thrombolysis and endovascular thrombectomy (EVT). In their new study [1], Novak et al. have shown that a shorter time from stroke onset to groin puncture is associated with better recanalisation. This underlines the crucial importance of time in stroke care.
\end{abstract}

Key words: Ischaemic stroke, thrombectomy, recanalisation

(Neurol Neurochir Pol 2020; 54 (2): 104-105)

Stroke is a leading cause of mortality and morbidity worldwide. The rate of hospitalisation for recurrent stroke in the Silesian province of Poland was 22.2\% between 2009 and 2015 [2]. Modern neuroimaging allows for the rapid selection of suitable candidates for acute reperfusion therapy in order to reduce disability after stroke. In addition to intravenous thrombolysis, at least seven randomised clinical trials have demonstrated the safety and effectiveness of EVT for the treatment of large-vessel occlusive ischaemic stroke within six hours of symptom onset. In 2018, two multicentre RCTs, DAWN and DEFUSE-3, similarly showed that EVT is also superior to standard medical treatment alone from six up to 24 hours $[3,4]$.

Although the stroke community has welcomed the addition of EVT to intravenous thrombolysis in acute ischaemic stroke treatment to reduce disability, we must not overlook those patients who do not respond to intravenous thrombolysis or EVT. At least $50 \%$ of patients do not achieve functional independence despite undergoing EVT, and about $15 \%$ of these patients do not survive [5]. Similarly, intravenous thrombolysis alone opens about $50 \%$ of occluded arteries but $14-34 \%$ of these re-occlude within two hours, leading to worse clinical outcomes [6]. In a large academic stroke cohort in Poland, Wiącek et al. reported that only $21 \%$ of patients presenting with acute stroke symptoms allowed initiation of endovascular procedures within six hours of symptom onset [7].

In order to continue to improve stroke outcome in patients undergoing reperfusion therapy, we must investigate factors that attenuate success in recanalisation.
'Time is brain' is the mantra in acute stroke. Even in an extended time window, the collateral vessels that protect brain tissue from ischaemia will fail without reperfusion. Nowak et al. have conducted an important single-centre study in Poland involving 223 consecutive patients presenting with large-vessel occlusion who underwent mechanical thrombectomy to evaluate the relationship between time of stroke onset and groin puncture (SO-GP) [1]. Mean admission NIHSS (National Institutes of Health Stroke Scale) was $15.6 \pm 5.3$. Intravenous thrombolysis was administered in $68.6 \%$ of patients. At least two thrombectomy passes were required in $20.6 \%$ of cases. Median SO-GP time was 240 minutes (IQR range: 180-305 minutes). Complete or near-complete recanalisation was achieved in $70.4 \%$ of patients. The authors looked for predictors associated with recanalisation. After adjusting for covariates in multivariable models, they found that shorter SO-GP is associated with a higher rate of recanalisation.

These findings resonate with a recent meta-analysis of individual patient data from seven randomised trials of the Highly Effective Reperfusion Using Multiple Endovascular Devices (HERMES) group involving 728 patients. This reported a significant $22 \%$ relative reduction per additional hour between stroke admission and groin puncture, and a $26 \%$ relative reduction per additional hours between imaging and puncture. These findings confirm the great importance of time in patients presenting with large-vessel occlusion of the internal carotid artery and/or the middle cerebral artery [8]. 
This study uniquely reported stroke outcomes in Polish populations, which were not represented in previous trials. The positive findings from the study underline the importance of time to groin puncture. Time from stroke onset to arrival in the emergency room can depend on an individual's recognition of potential stroke, because the most common reason for delayed presentation is a failure to recognise symptoms.

Once patients arrive at hospital, time-to-imaging, time-to-groin-puncture, and time-to-recanalisation all depend on the system of care in place at the particular hospital.

The authors of this study have undoubtedly demonstrated that time is of great importance in acute stroke care.

\section{References:}

1. Nowak K, Derbisz J, Jagiełła J, et al. Time from stroke onset to groin puncture affects rate of recanalisation after mechanical thrombectomy: a real-life single centre experience. Neurol Neurochir Pol. 2020, doi: $10.5603 /$ pjnns.a2020.0024.

2. Łabuz-Roszak B, Skrzypek M, Starostka-Tatar A, et al. Epidemiological analysis of hospitalisations due to recurrent stroke in the Silesian Province, Poland, between 2009 and 2015. Neurol Neurochir Pol. 2019; 53(4): 277290, doi: 10.5603/PJNNS.a2019.0034, indexed in Pubmed: 31441494.

3. Albers GW, Marks MP, Kemp S, et al. Defuse 3 Investigators. Thrombectomy for Stroke at 6 to 16 Hours with Selection by Perfusion
Imaging. N Engl J Med. 2018; 378(8): 708-718, doi: 10.1056/NEJMoa1713973, indexed in Pubmed: 29364767.

4. Nogueira RG, Jadhav AP, Haussen DC, et al. Dawn Trial Investigators. Thrombectomy 6 to 24 Hours after Stroke with a Mismatch between Deficit and Infarct. N Engl J Med. 2018; 378(1): 11-21, doi: 10.1056/ NEJMoa1706442, indexed in Pubmed: 29129157.

5. Goyal M, Menon BK, van Zwam WH, et al. Hermes collaborators. Endovascular thrombectomy after large-vessel ischaemic stroke: a meta-analysis of individual patient data from five randomised trials. Lancet. 2016; 387(10029): 1723-1731, doi: 10.1016/S0140-6736(16)00163-X, indexed in Pubmed: 26898852.

6. Alexandrov AV, Molina CA, Grotta JC, et al. Clotbust Investigators. Ultrasound-enhanced systemic thrombolysis for acute ischemic stroke. N Engl J Med. 2004; 351(21): 2170-2178, doi: 10.1056/NEJMoa041175, indexed in Pubmed: 15548777.

7. Wiącek M, Kaczorowski R, Sieczkowski B, et al. Mechanical thrombectomy: Determining the proportion of eligible acute ischemic stroke patients in the cohort of single academic stroke center. Neurol Neurochir Pol. 2018; 52(3): 359-363, doi: 10.1016/i.pinns.2017.12.010, indexed in Pubmed: 29331206.

8. Bourcier R, Goyal M, Liebeskind DS, et al. Hermes Trialists Collaboration. Association of Time From Stroke Onset to Groin Puncture With Quality of Reperfusion After Mechanical Thrombectomy: A Meta-analysis of Individual Patient Data From 7 Randomized Clinical Trials. Jama Neurol. 2019; 76(4): 405-411, doi: 10.1001/jamaneurol.2018.4510, indexed in Pubmed: $\underline{30667465}$. 